\title{
Efeito das posições dos enxertos na estabilidade da artroplastia total de quadril com diferentes tipos de encurtamento subtrocantérico*
}

\section{The Effect of Graft Positions on the Stability of Total Hip Arthroplasty with Different Types of Subtrochanteric Shortening}

\author{
Ethem Aytac Yazar ${ }^{1,2,3}$ Ozgun Karakus 1,2,30 Baransel Saygi1,2,3 \\ ${ }^{1}$ Departamento de Cirurgia Ortopédica, Fatih Sultan Mehmet Training \\ and Research Hospital, Duzici City Hospital, Osmaniye, Turquia \\ 2 Departamento de Cirurgia Ortopédica, Fatih Sultan Mehmet Training and \\ Endereço para correspondência Ozgun Karakus, MD, Alparslan \\ Turkes st. Consept Nigde bloco n. A-24 Niðde, Turquia \\ (e-mail: ozgunkarakus@hotmail.com).
} Research Hospital, Omer Halis Demir University Hospital, Nigde, Turquia

${ }^{3}$ Departamento de Cirurgia Ortopédica, Maltepe University and Fatih Sultan Mehmet Training and Research Hospital, Istanbul, Turquia

Rev Bras Ortop 2019;54:465-470.

\section{Resumo}

Palavras-chave

- osteotomia

- transplante ósseo

- coxartrose

- artroplastia de quadril

- luxação do quadril
Objetivo O objetivo do presente estudo é investigar a estabilidade biomecânica de diferentes tipos de osteotomias subtrocantéricas e posições de enxertos em coxartroses displásicas que necessitam de artroplastia total de quadril com osteotomia de encurtamento, e determinar o tipo de osteotomia e a posição do enxerto que são mais eficazes. Método Modelos de fêmur (sawbones) foram usados para comparar os tipos de osteotomia de encurtamento femoral (transversal, oblíqua e em degrau de escada [step-cut]). Os enxertos em haste, preparados do lado da osteotomia subtrocantérica, foram fixados em diferentes posições (ântero-lateral, medial-lateral e ântero-posterior). A fixação dos enxertos foi feita com 2 cabos de aço (de 2,0 mm de largura) de mesma resistência. Os valores de falha dos fêmures compostos para cargas axiais e rotacionais foram registrados.

Resultados Do ponto de vista biomecânico, não houve diferenças estatisticamente significativas entre os tipos de osteotomia de encurtamento subtrocantérico femoral e as posições dos enxertos em haste aplicados.

Conclusão Não houve superioridade entre os tipos de osteotomia de encurtamento subtrocantérico femoral quanto à estabilidade. Além disso, em relação à resistência ao estresse, os resultados obtidos com diferentes posições das hastes foram similares. Assim, acreditamos que o método mais indicado é aquele em que o cirurgião é experiente e cuja aplicação é mais fácil.
Estudo realizado no Departamento de Cirurgia Ortopédica, Fatih Sultan Mehmet Training and Research Hospital, Omer Halis Demir University Hospital, Nigde, Turquia.

(D) Ozgun Karakus's ORCID is https://orcid.org/0000-0002-7430-1768. recebido

29 de Setembro de 2018

aceito

06 de Novembro de 2018
DOI https://doi.org/

10.1055/s-0039-1694022. ISSN 0102-3616.
Copyright $\odot 2019$ by Sociedade Brasileira License terms de Ortopedia e Traumatologia. Published by Thieme Revnter Publicações Ltda, Rio de Janeiro, Brazil 


\begin{abstract}
Keywords

- osteotomy

- bone transplantation

- coxarthrosis

- arthroplasty, replacement, hip

- hip dislocation

Objective The aim of the present study is to investigate the biomechanical stability of different subtrochanteric osteotomy types and graft positions in dysplastic coxarthrosis that require total hip arthroplasty with shortening osteotomy, as well as to find out the most effective osteotomy type and graft position.

Method Femur sawbones were used to compare different types of femoral shortening osteotomy (transverse, oblique, and step-cut). Strut grafts, which were prepared at the side of the subtrochanteric shortening osteotomy, were fixed in different positions (anterior-lateral, medial-lateral, and anterior-posterior). The fixation of the strut grafts was performed using two steel cables (with $2.0 \mathrm{~mm}$ of thickness) with the same strength. The failure values of composite femurs were recorded for axial and rotational loadings.

Results Biomechanically, there were no statistically significant differences between the types of femoral subtrochanteric shortening osteotomy and the positions of the applied strut graft.

Conclusion No superiority was observed between the types of femoral subtrochanteric shortening osteotomy regarding stability. Additionally, against stress, similar results were gathered for different strut graft positions. In conclusion, we believe that using the method in which the surgeon is experienced and that is easier to be applied would be the best choice.
\end{abstract}

\section{Introdução}

As articulações coxofemorais são uma estrutura anatômica conectada aos membros inferiores do corpo para que a postura seja ereta e o movimento, equilibrado. Essas articulações são formadas para modificações ilimitadas e amplificações de movimentos básicos, como força, movimento, caminhada, corrida, salto e subida. ${ }^{1}$ A articulação coxofemoral é a mais sobrecarregada do corpo humano. ${ }^{2}$ Por isso, há risco de desenvolvimento natural de artrite degenerativa ao longo da vida funcional. ${ }^{3}$

Em uma articulação coxofemoral com artrite degenerativa, 0 principal objetivo terapêutico é o alívio da dor e a normalização da série de movimentos articulares. Atualmente, não é possível restaurar por completo a articulação coxofemoral, afetada por muitas razões e desgastada devido à natureza fisiológica da estrutura da cartilagem, à sua estrutura natural. Métodos como osteotomias, artroplastias de ressecção e artrodeses do quadril, projetados para equilibrar a distribuição articular de cargas e aliviar a dor, ainda são utilizados quando necessário. ${ }^{4,5} \mathrm{~A}$ artroplastia total do quadril é uma solução alternativa. A princípio cimentadas, as artroplastias totais do quadril têm sido progressivamente substituídas por sistemas híbridos e não cimentados. ${ }^{6-8}$

A displasia coxofemoral do desenvolvimento envolve uma ampla gama de patologias que vão desde uma simples instabilidade do quadril por frouxidão capsular até a luxação completa, em que a cabeça do fêmur está totalmente fora do acetábulo. ${ }^{9}$ Casos que não podem ser detectados na infância podem causar osteoartrite do quadril em adultos jovens. ${ }^{10}$

Em pacientes sem diagnóstico e tratamento precoces, e em adultos jovens tratados de maneira inadequada, as osteotomias acetabulares e femorais são realizadas para prevenir osteoartrite e outras patologias que podem se desenvolver secundariamente à displasia. ${ }^{3,11,12}$ Apesar dessas intervenções cirúrgicas, a degeneração articular e a osteoartrite coxofemoral ainda podem ocorrer. Em pacientes com dor e limitação funcional devido à osteoartrite, os resultados da artroplastia total do quadril são satisfatórios. ${ }^{13}$

Este estudo tem como objetivo a comparação biomecânica do efeito das posições dos enxertos e das técnicas de osteotomia subtrocantérica na estabilidade primária da artroplastia total do quadril com necessidade de encurtamento.

\section{Materiais e Métodos}

A adequação deste estudo foi aprovada pelo Comitê de Ética do Fatih Sultan Mehmet Education and Training Hospital.

Um total de 63 fêmures compostos (sawbones, item número 1130, esquerdo médio, TST AŞ, İstanbul-Turkey) foram usados em cada experimento de carga. Os fêmures foram divididos em três grupos de acordo com os tipos de osteotomia de encurtamento femoral: tranversal (grupo TO), oblíqua (grupo 00) e em degrau de escada (step-cut; grupo SO). Além disso, cada grupo também foi dividido em três subgrupos de acordo com as posições dos enxertos; ânteroposterior (subgrupo AP), medial-lateral (subgrupo ML) e ântero-lateral (subgrupo AL).

A ressecção óssea segmentar de $4 \mathrm{~cm}$ (encurtamento) foi feita na região subtrocantérica de cada modelo femoral de acordo com o tipo de osteotomia do grupo. Os lados da osteotomia foram calibrados com um paquímetro (Astor Vernier, Calipar, China) e marcados com caneta. Após o término da osteotomia, o osso removido foi seccionado em duas partes iguais em sentido vertical. Esses dois enxertos foram colocados em posição ântero-posterior, medial-lateral 


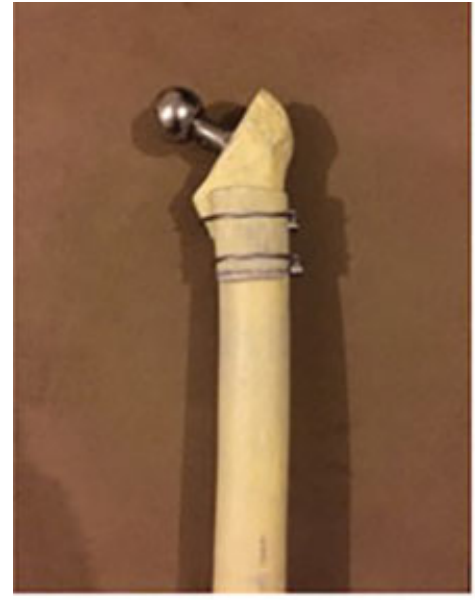

Ântero-posterior

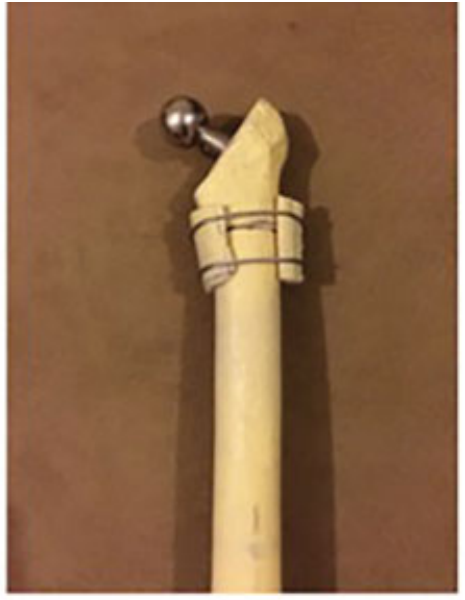

Medial-lateral

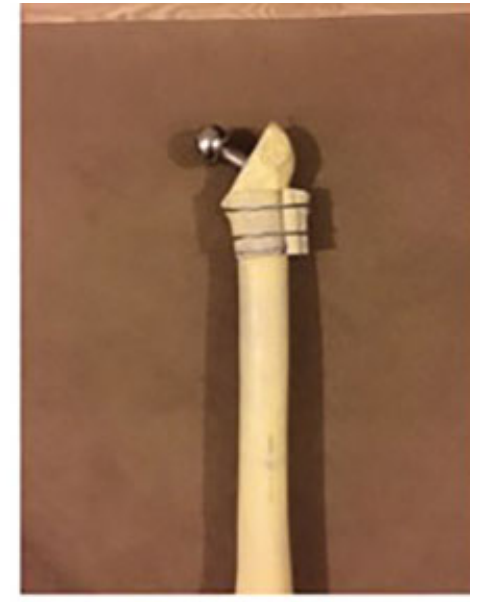

Ântero-lateral

Fig. 1 Posição dos enxertos: ântero-posterior, medial-lateral e ântero-lateral.

e ântero-lateral, com centralização no lado da osteotomia, e fixados com cabos em volta de cada osso composto (-Fig. 1). Este procedimento foi repetido de forma semelhante em cada osso de acordo com o tipo de osteotomia do grupo.

Para a fixação, 2 cabos ósseos (Accord cable system, n. 7136-0005, Smith and Nephew, Memphis, TN, EUA) foram usados em cada enxerto e submetidos à tensão de 50 libras por polegada. Uma haste cônica estriada (cone Wagner, tamanho número 21/135 Zimmer, Warsaw, IN, EUA) foi implantada como prótese femoral em cada osso.

Cargas axiais e rotacionais de $2.500 \mathrm{~N}$ e $200 \mathrm{~N}$, respectivamente, foram aplicadas nos ossos compostos. Os experimentos foram realizados em um laboratório biomecânico com dispositivo de teste (célula de carga Instron, SHIMADZU 10KN AGS-J; made in Kyoto, Japan, 8802). A taxa de carga foi de $5 \mathrm{~mm}$ por minuto em todas as amostras (taxa de dados de $50 \mathrm{~ms} / 200 \mathrm{~Hz})^{14}$

\section{Resultados}

Todas as amostras foram testadas até a falha. Os valores do estresse de rompimento de cada amostra foram medidos. Em todas, o material composto do fêmur foi fraturado a partir da extremidade distal do componente da prótese femoral. Os resultados das cargas axiais e rotacionais são mostrados nas - Tabelas $\mathbf{1}$ e $\mathbf{2}$.

Não houve diferença estatisticamente significativa entre os tipos de osteotomia quando o enxerto foi colocado em posição ântero-posterior, medial-lateral ou ântero-lateral.

Não houve diferença significativa entre os tipos de osteotomia ou entre as posições dos enxertos entre os grupos ou dentro de cada grupo.

A análise estatística foi realizada usando o programa Statistical Package for the Social Sciences (SPSS, SPSS, Inc., Chicago, IL, EUA), versão 16.0. Os dados foram analisados por meio de estatística descritiva (média, desvio padrão, mediana, frequência, porcentagem, valor mínimo e valor máximo). Os testes de Kruskal-Wallis e U de Mann-Whitney foram utilizados para a comparação entre e dentro dos três grupos.

\section{Discussão}

Em 1973, Charnley e Feagin relataram que a artroplastia total do quadril deve ser evitada em quadris displásicos, especialmente em luxações de tipo 3 e 4 de Crowe. ${ }^{15}$ No entanto, com o desenvolvimento de novos tipos e técnicas de prótese, a artroplastia total do quadril começou a ser aplicada em indivíduos mais jovens, mesmo em casos de displasia. ${ }^{16,17}$

No tratamento cirúrgico da coxartrose decorrente da displasia do desenvolvimento, o abaixamento do acetábulo à sua altura verdadeira é amplamente aceito. Isso ajuda a reduzir a força de reação articular, aumenta o braço de alavanca do abdutor, e, consequentemente, melhora a função da marcha e prolonga a sobrevida mecânica do componente acetabular. ${ }^{18}$

A aplicação total da prótese de quadril na coxartrose secundária à displasia do desenvolvimento pode diferir significativamente da aplicação na osteoartrite primária. A migração proximal do fêmur cria alterações anatômicas e biomecânicas nos tecidos ósseos e moles que tornam a artroplastia tecnicamente desafiadora e mais propensa a complicações. ${ }^{19}$ Muitos autores enfatizam que a osteotomia de subtração femoral deve ser realizada para prevenir complicações neurovasculares em quadris de tipo 3 e 4 de Crowe, que não são submetidos à redução. ${ }^{19-22}$

Dois tipos principais de osteotomia de subtração femoral têm sido descritos tecnicamente na literatura. ${ }^{20,21} \mathrm{O}$ primeiro consiste na ressecção proximal do fêmur e deslocamento distal do trocanter maior, enquanto o segundo é o encurtamento metafisário subtrocantérico sem osteotomia do trocânter maior.

Sem encurtamento do fêmur, é quase impossível levar a cabeça do osso à altura real do acetábulo. Por isso, a subtração é o procedimento padrão nestes pacientes. Durante a revisão das séries clínicas da literatura, observou-se que os autores optaram pela técnica de osteotomia com base em sua própria experiência clínica. $O$ encurtamento médio variou de $3,1 \mathrm{~cm}$ a $5 \mathrm{~cm}$ em várias séries. ${ }^{23,24}$ No presente estudo, 0 valor de $4 \mathrm{~cm}$ foi usado considerando esses valores. ${ }^{14}$

O propósito de usar um fêmur composto é montar experimentos no mais alto nível, sem a dificuldade de armazenamento 
Tabela 1 Distribuição dos valores de falha dos compostos femorais submetidos a cargas axiais de acordo com o grupo (N/mm)

\begin{tabular}{|c|c|c|c|c|c|c|c|}
\hline & & \multicolumn{3}{|c|}{ Carga de falha femoral } & \multirow{2}{*}{$\begin{array}{l}\text { Valor de } p \\
\text { ântero-posterior/ } \\
\text { medial-lateral }\end{array}$} & \multirow{2}{*}{$\begin{array}{l}\text { Valor de } p \\
\text { ântero-posterior| } \\
\text { ântero-lateral }\end{array}$} & \multirow{2}{*}{$\begin{array}{l}\text { Valor de } p \\
\text { medial-lateral } \\
\text { ântero-lateral }\end{array}$} \\
\hline & & Ântero-Posterior & Medial-Lateral & Ântero-Lateral & & & \\
\hline \multirow[t]{3}{*}{ Grupo TO } & $\mathrm{N}$ & 7 & 7 & 7 & \multirow[t]{3}{*}{0,565} & \multirow[t]{3}{*}{0,949} & \multirow[t]{3}{*}{0,749} \\
\hline & Média \pm DP & $827,61 \pm 51,81$ & $842,11 \pm 48,14$ & $828,38 \pm 34,66$ & & & \\
\hline & Mín-Máx & $761,82-912,69$ & $790,61-910,42$ & $788,04-875,38$ & & & \\
\hline \multirow[t]{3}{*}{ Grupo 00} & $\mathrm{~N}$ & 7 & 7 & 7 & \multirow[t]{3}{*}{0,749} & \multirow[t]{3}{*}{0,848} & \multirow[t]{3}{*}{0,949} \\
\hline & Média \pm DP & $837,41 \pm 38,19$ & $850,24 \pm 49,86$ & $842,11 \pm 52,68$ & & & \\
\hline & Mín-Máx & $783,59-886,43$ & $780,73-907,81$ & $797,38-916,93$ & & & \\
\hline \multirow[t]{3}{*}{ Grupo SO } & $\mathrm{N}$ & 7 & 7 & 7 & \multirow[t]{3}{*}{0,277} & \multirow[t]{3}{*}{0,482} & \multirow[t]{3}{*}{0,337} \\
\hline & Média \pm DP & $838,71 \pm 48,15$ & $853,53 \pm 33,99$ & $826,06 \pm 47,66$ & & & \\
\hline & Mín-Máx & $791,82-913,43$ & $817,54-917,75$ & $765,61-881,03$ & & & \\
\hline \multicolumn{2}{|l|}{${ }^{\mathrm{a} V a l o r ~ d e ~} p$} & 0,872 & 0,763 & 0,708 & & & \\
\hline \multicolumn{2}{|c|}{$\begin{array}{l}\text { balor de } p \\
\text { (grupo 1-grupo 2) }\end{array}$} & 0,655 & 0,848 & 0,565 & & & \\
\hline \multicolumn{2}{|c|}{$\begin{array}{l}\text { balor de } p \\
\text { (grupo 1-grupo 3) }\end{array}$} & 0,655 & 0,337 & 0,949 & & & \\
\hline \multicolumn{2}{|c|}{$\begin{array}{l}\text { balor de } p \\
\text { (grupo 2- grupo 3) }\end{array}$} & 0,949 & 0,949 & 0,406 & & & \\
\hline
\end{tabular}

Notas: ${ }^{\mathrm{a}}$ Teste de Kruskal-Wallis; ${ }^{\mathrm{b}}$ Teste U de Mann-Whitney; ${ }^{p} \mathrm{p}<0.05$.

Abreviaturas: DP, desvio padrão; Máx, máximo; Mín, mínimo; OO, osteotomia oblíqua; SO, osteotomia step-cut; TO, osteotomia transversal.

Tabela 2 Distribuição dos valores de falha dos compostos femorais submetidos a cargas rotacionais de acordo com o grupo (N/mm)

\begin{tabular}{|c|c|c|c|c|c|c|c|}
\hline & & \multicolumn{3}{|c|}{ Carga de falha femoral } & \multirow{2}{*}{$\begin{array}{l}\text { Valor de } p \\
\text { ântero-posterior/ } \\
\text { medial-lateral }\end{array}$} & \multirow{2}{*}{$\begin{array}{l}\text { Valor de } p \\
\text { ântero-posterior/ } \\
\text { ântero-lateral }\end{array}$} & \multirow{2}{*}{$\begin{array}{l}\text { Valor de } p \\
\text { medial-lateral } \\
\text { ântero-lateral }\end{array}$} \\
\hline & & Ântero-Posterior & Medial-Lateral & Ântero-Lateral & & & \\
\hline \multirow[t]{3}{*}{ Grupo TO } & $\mathrm{N}$ & 7 & 7 & 7 & \multirow[t]{3}{*}{0,87} & \multirow[t]{3}{*}{0,99} & \multirow[t]{3}{*}{0,75} \\
\hline & Média \pm DP & $54,87 \pm 3,43$ & $55,64 \pm 3,18$ & $54,83 \pm 2,28$ & & & \\
\hline & Mín-Máx & $50,49-60,55$ & $52,21-60,19$ & $52,14-57,96$ & & & \\
\hline \multirow[t]{3}{*}{ Grupo 00} & $\mathrm{~N}$ & 7 & 7 & 7 & \multirow[t]{3}{*}{0,81} & \multirow[t]{3}{*}{0,87} & \multirow[t]{3}{*}{0,98} \\
\hline & Média \pm DP & $55,53 \pm 2,53$ & $56,18 \pm 3,29$ & $56,14 \pm 3,49$ & & & \\
\hline & Mín-Máx & $51,84-58,70$ & $51,75-60,22$ & $52,66-60,63$ & & & \\
\hline \multirow[t]{3}{*}{ Grupo SO } & $\mathrm{N}$ & 7 & 7 & 7 & \multirow[t]{3}{*}{0,49} & \multirow[t]{3}{*}{0,69} & \multirow[t]{3}{*}{0,42} \\
\hline & Média \pm DP & $55,51 \pm 3,18$ & $56,40 \pm 2,23$ & $54.77 \pm 3.16$ & & & \\
\hline & Mín-Máx & $52,39-60,50$ & $54,00-60,68$ & $50.74-58.43$ & & & \\
\hline \multicolumn{2}{|l|}{${ }^{a}$ Valor de $p$} & 0,96 & 0,88 & 0,78 & & & \\
\hline \multicolumn{2}{|c|}{$\begin{array}{l}\text { bValor de } p \\
\text { (grupo 1- grupo 2) }\end{array}$} & 0,69 & 0,88 & 0,61 & & & \\
\hline \multicolumn{2}{|c|}{$\begin{array}{l}\text { bValor de } p \\
\text { (grupo 1- grupo 3) }\end{array}$} & 0,66 & 0,53 & 0,96 & & & \\
\hline \multicolumn{2}{|c|}{$\begin{array}{l}{ }^{\mathrm{b}} \text { Valor de } p \\
\text { (grupo 2- grupo 3) }\end{array}$} & 0,99 & 0,96 & 0,52 & & & \\
\hline
\end{tabular}

Notas: ${ }^{a}$ Teste de Kruskal-Wallis; ${ }^{\mathrm{b}}$ Teste U de Mann-Whitney; ${ }^{*} p<0.05$.

Abreviaturas: DP, desvio padrão; Máx, máximo; Mín, mínimo; OO, osteotomia oblíqua; SO, osteotomia step-cut; TO, osteotomia transversal.

de espécimes cadavéricos e com a possibilidade de padronização de condições, como materiais, e a adequação comercial.

Há vários estudos sobre o efeito das forças de reação articular e da distribuição da pressão na superfície articular do quadril durante o apoio unipodal e em caminhadas. ${ }^{25,26}$ No estudo atual, a carga axial escolhida foi de $2.500 \mathrm{~N}$, o equivalente aproximado a 3,5 vezes o peso corpóreo adulto médio. ${ }^{14}$ A transferência de carga na linha de osteotomia 
pode afetar negativamente a fratura em alguns pacientes submetidos à osteotomia subtrocantérica. ${ }^{26,27}$ Por isso, a linha de osteotomia precisa ser estabilizada. Acredita-se que a osteotomia transversal tenha baixa estabilidade rotacional. No entanto, resultados semelhantes foram relatados em técnicas difíceis (como step-cut), consideradas de alta estabilidade rotacional. ${ }^{18,24,28-30}$

Muitos estudos biomecânicos foram realizados com osteotomias trocantéricas e subtrocantéricas, além de osteotomias pélvicas relacionadas a artroplastias totais do quadril. No entanto, de acordo com o conhecimento atual, não há estudos sobre a posição do enxerto na artroplastia total do quadril combinada à osteotomia de encurtamento subtrocantérico. No presente estudo, os métodos de osteotomia e a relação de estabilidade conforme as posições dos enxertos na linha de osteotomia foram investigados. Não houve diferença estatisticamente significativa entre os métodos de osteotomia e o posicionamento dos enxertos.

Gotze et $a^{30}$ investigaram a necessidade de fixação extramedular na osteotomia transversa de encurtamento subtrocantérico. Os autores observaram que a estabilização intramedular está relacionada à geometria transversal do componente femoral, que também está relacionada à estabilidade rotacional distal. Também sugeriram que a osteotomia step-cut ou o uso de enxertos em hastes e a fixação de cabos não são necessários. Por outro lado, embora se acredite que as propriedades estruturais dos implantes empregados na artroplastia sejam importantes na fixação da osteotomia, ${ }^{29}$ muitos autores recomendam a fixação de enxertos e cabos, a combinação placacabo, ou a osteossíntese com parafusos e placas para melhorar a estabilidade. ${ }^{23,27,30}$ Além disso, Muratli et al ${ }^{14}$ verificaram que não houve diferença entre os tipos de osteotomias em termos de estabilidade, mas observaram que o enxerto na linha de osteotomia aumentou a estabilidade de forma individual.

Nossa hipótese é a de que o posicionamento ântero-lateral do enxerto na linha de osteotomia é mais fácil, e que não há diferença biomecânica em termos de estabilização em conjunto.

O presente trabalho pode apresentar algumas limitações. Como os testes foram realizados in vitro, não há efeitos de estruturas musculares e ligamentares. Estudos de análise de elementos finitos ou com cadáveres envolvendo esses parâmetros podem ser projetados para esse fim.

\section{Conclusão}

No presente estudo, em casos de coxartrose displásica com necessidade de artroplastia total do quadril com encurtamento, os métodos de osteotomia e posicionamento dos enxertos foram investigados e comparados quanto à estabilidade antes da consolidação da linha óssea na linha de osteotomia. Descobriu-se que diferentes tipos de osteotomia subtrocantérica e locais de enxerto apresentaram resistência e estabilidade similares.

Como conclusão, devido à ausência de superioridade das técnicas existentes, sugere-se a aplicação do método considerado mais fácil pelo cirurgião ou com o qual o profissional tenha maior experiência.

\section{Conflitos de Interesse}

Os autores declaram não haver conflitos de interesse.

\section{Referências}

1 Ganey TM, Ogden JA. Pre and postnatal development of the hip. In: Callaghan JJ, Rosenberg AG, Rubash HE, editores. The adult hip. 2. ed. Filadélfia: Lippincott Williams \& Wilkins; 2007:35

2 Barrack RL, Newland CC. Uncemented total hip arthroplasty with superior acetabular deficiency. Femoral head autograft technique and early clinical results. J Arthroplasty 1990;5(02):159-167

3 Steel HH. Triple osteotomy of the innominate bone. J Bone Joint Surg Am 1973;55(02):343-350

4 Wedge $\mathrm{JH}$, Wasylenko MJ. The natural history of congenital disease of the hip. J Bone Joint Surg Br 1979;61-B(03):334-338

5 Trousdale RT, Cabanela ME. Lessons learned after more than 250 periacetabular osteotomies. Acta Orthop Scand 2003;74(02):119-126

6 Charnley J. Anchorage of the femoral head prosthesis to the shaft of the femur. J Bone Joint Surg Br 1960;42-B:28-30

7 Bobyn JD, Pilliar RM, Cameron HU, Macnab I. Porous surfaced layered prosthetic devices. J Biomed Eng 1975;10(04):126-131

8 Bonfiglio M, Voke EM. Aseptic necrosis of the femoral head and nonunion of the femoral neck. J Bone Joint Surg Am 1968;50(01): 48-66

9 Crowe JF, Mani VJ, Ranawat CS. Total hip replacement in congenital dislocation and dysplasia of the hip. J Bone Joint Surg Am 1979;61(01):15-23

10 Doğan A, Zorer G, Ozer UE. [Treatment of acetabular dysplasia by triple pelvic osteotomy and its short-term results]. Acta Orthop Traumatol Turc 2007;41(05):355-366

11 Tönnis D, Arning A, Bloch M, Heinecke A, Kalchschmidt K. Triple pelvic osteotomy. J Pediatr Orthop B 1994;3:54-67

12 Ganz R, Klaue K, Vinh TS, Mast JW. A new periacetabular osteotomy for the treatment of hip dysplasias. Technique and preliminary results. Clin Orthop Relat Res 1988;(232):26-36

13 Akman Ş, Şen C, Şener N, Tözün R. Doğuştan kalça çıkığı ve displazisinde total kalça artroplastisi. Acta Orthop Traumatol Turc 2000;34:176-182

14 Muratli KS, Karatosun V, Uzun B, Celik S. Subtrochanteric shortening in total hip arthroplasty: biomechanical comparison of four techniques. J Arthroplasty 2014;29(04):836-842

15 Charnley J, Feagin JA. Low-friction arthroplasty in congenital subluxation of the hip. Clin Orthop Relat Res 1973;(91):98-113

16 Harris WH. Total hip arthroplasty in the management of the congenital hip dislocation. In: Callaghan JJ, Rosenberg AG, Rubash HE, editores. The adult hip. Filadélfia: Lippincott-Rawen; 1988: 1651-1682

17 Chougle A, Hemmady MV, Hodgkinson JP. Long-term survival of the acetabular component after total hip arthroplasty with cement in patients with developmental dysplasia of the hip. J Bone Joint Surg Am 2006;88(01):71-79

18 Kiliçoğlu OI, Türker M, Akgül T, Yazicioğlu O. Cementless total hip arthroplasty with modified oblique femoral shortening osteotomy in Crowe type IV congenital hip dislocation. J Arthroplasty 2013;28(01):117-125

19 Symeonides PP, Pournaras J, Petsatodes G, Christoforides J, Hatzokos I, Pantazis E. Total hip arthroplasty in neglected congenital dislocation of the hip. Clin Orthop Relat Res 1997;(341):55-61

20 Dunn HK, Hess WE. Total hip reconstruction in chronically dislocated hips. J Bone Joint Surg Am 1976;58(06):838-845

21 Paavilainen T, Hoikka V, Solonen KA. Cementless total replacement for severely dysplastic or dislocated hips. J Bone Joint Surg Br 1990; 72(02):205-211

22 Paavilainen T, Hoikka V, Paavolainen P. Cementless total hip arthroplasty for congenitally dislocated or dysplastic hips. Technique for replacement with a straight femoral component. Clin Orthop Relat Res 1993;(297):71-81 
23 Togrul E, Ozkan C, Kalaci A, Gülșen M. A new technique of subtrochanteric shortening in total hip replacement for Crowe type 3 to 4 dysplasia of the hip. J Arthroplasty 2010;25(03): 465-470

24 Reikeraas O, Lereim P, Gabor I, Gunderson R, Bjerkreim I. Femoral shortening in total arthroplasty for completely dislocated hips: 3-7 year results in 25 cases. Acta Orthop Scand 1996;67(01):33-36

25 Davy DT, Kotzar GM, Brown RH, et al. Telemetric force measurements across the hip after total arthroplasty. J Bone Joint Surg Am 1988;70(01):45-50

26 Lim LA, Carmichael SW, Cabanela ME. Biomechanics of total hip arthroplasty. Anat Rec 1999;257(03):110-116

27 Bruce WJ, Rizkallah SM, Kwon YM, Goldberg JA, Walsh WR. A new technique of subtrochanteric shortening in total hip arthroplasty: surgical technique and results of 9 cases. J Arthroplasty 2000;15 (05):617-626

28 Sun JY, Wei L. Subtrochanteric shortening with overlapping femoral resection in total hip arthroplasty for Crowe type IV adult dislocation of the hip. Orthop Surg 2009;1(03):207-211

29 Bernasek TL, Haidukewych GJ, Gustke KA, Hill O, Levering M. Total hip arthroplasty requiring subtrochanteric osteotomy for developmental hip dysplasia: 5- to 14-year results. J Arthroplasty 2007;22(06, Suppl 2):145-150

30 Götze C, Winkelmann W, Gosheger G, Rödl R. [Is there a need of an additional extramedullary fixation in transverse subtrochanteric shortening in primary total hip arthroplasty for patients with severe hip dysplasia? Short-term experience in seven patients with congenital dislocation]. Z Orthop Unfall 2007;145(05):568-573 